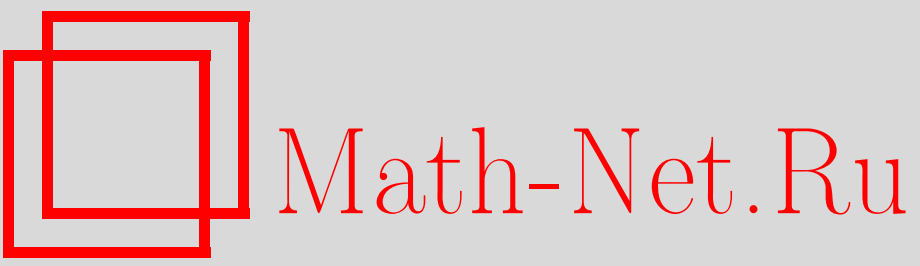

Е. М. Матвеев, Об индексе мультипликативных групп алгебраических чисел, Матем. сб., 2005, том 196, номер 9, 59-70

DOI: https://doi.org/10.4213/sm1420

Использование Общероссийского математического портала Math-Net.Ru подразумевает, что вы прочитали и согласны с пользовательским соглашением

http://www.mathnet.ru/rus/agreement

Параметры загрузки:

IP : 54.80 .73 .141

26 апреля 2023 г., 15:12:18 


\author{
Е. М. Матвеев
}

\title{
Об индексе мультипликативных групп алгебраических чисел
}

\begin{abstract}
Пусть в алгебраическом поле фиксированы ненулевые числа с некоторыми линейно независимьми значениями логарифмов. Рассматривается множество чисел этого поля, логарифомы которых рационально выражаются через логарифмы данных чисел. Соответствующие векторы коэффициентов образуют решетку, содержашую стандартную целочисленную решетку в качестве подрешетки конечного индекса. В работе дается усиление верхней оценки этого индекса в терминах расширенных логариффмических высот участвующих чисел. Попутно это дает оценку коэффициентов в целочисленных линейных соотношениях между логарифмами алгебраических чисел.
\end{abstract}

Библиография: 13 названий.

\section{§1. Введение}

В 1933 г. Д. Лемер [1] выдвинул гипотезу, что так называемая мера Малера целых алгебраических чисел, отличных от нуля и корней из единицы, ограничена снизу абсолютной константой $c>1$. Напомним, что для числа $\alpha$, являюшегося корнем неприводимого (в том числе примитивного) многочлена

$$
a_{0}\left(X-\alpha^{(1)}\right) \cdots\left(X-\alpha^{(d)}\right)=a_{0} X^{d}+\cdots+a_{d-1} X+a_{d} \in \mathbb{Z}[X], \quad a_{0} \neq 0,
$$

мерой Малера называется выражение $\mathrm{M}(\alpha):=\left|a_{0}\right| \prod_{\sigma=1}^{d} \max \left\{1,\left|\alpha^{(\sigma)}\right|\right\}$. До сих пор гипотеза Лемера не доказана, хотя найденное им минимальное значение

$$
\mathrm{M}\left(\theta_{10}\right)=\theta_{10}=1.176 \ldots,
$$

где $\theta_{10}$ - корень многочлена

$$
x^{10}+x^{9}-x^{7}-x^{6}-x^{5}-x^{4}-x^{3}+x+1,
$$

не улучшено, несмотря на продолжительньй компьютерный поиск.

Из теоретических результатов следует упомянуть следуюшее.

Прежде всего, очевидно, что минимум следует искать среди целых алгебраических чисел $\left(a_{0}=1\right)$ и даже среди алгебраических единиц $\left(a_{d}= \pm 1\right)$, поскольку для других чисел мы имеем $\mathrm{M}(\alpha) \geqslant 2$. Далее, в 1971 г. К. Смит [2] доказал гипотезу Лемера для обширного класса чисел. Результат Смита гласит, что если $\alpha-$ не взаимное число, то $\mathrm{M}(\alpha) \geqslant \theta_{3}=1.324 \ldots$, где $\theta_{3}$ - корень многочлена $x^{3}-x-1$. (Взаимныц назьвается число $\alpha$, минимальный многочлен которого симметричен, равносильно, $\alpha$ и $1 / \alpha$ алгебраически сопряжены.) 
В 1971 г. Бланксби и Монтгомери [3] доказали общую оценку

$$
\mathrm{M}(\alpha) \geqslant 1+\frac{1}{52 d \ln (6 d)}
$$

В 1979 г. Е. Добровольский [4] получил лучшую на сегодня асимптотическую оценку

$$
\ln \mathrm{M}(\alpha) \geqslant(2-\varepsilon)\left(\frac{\ln \ln d}{\ln d}\right)^{3}, \quad d>d_{0}(\varepsilon) .
$$

В 1996 г. П. Вутье [5] заменил в оценке Добровольского величину $2-\varepsilon$ на $1 / 4$ для всех $d \geqslant 6$.

Другой задачей, имеющей важные приложения особенно в теории линейных форм от логарифмов алгебраических чисел, являются оценки с учетом величины $|\ln \alpha|$, где объединяющий параметр имеет вид

$$
A \geqslant \max \{\ln \mathrm{M}(\alpha),|\ln \alpha|\}
$$

Отметим, что здесь корни из единицы вида $\zeta_{q}=e^{2 \pi i / q}$ участвуют на равных с остальными числами, поскольку для них имеем $d=\operatorname{deg} \zeta_{q}=\varphi(q)$ - функция Эйлера, и

$$
\left|\ln \zeta_{q}\right|=\frac{2 \pi}{q} \geqslant \frac{\pi}{2 d \ln \ln (6 d)} .
$$

По этой причине утверждение, аналогичное гипотезе Лемера, неверно. Также заметим, что оценка $\left|\ln \zeta_{q}\right|$ имеет примерно такой же порядок малости, что и оценка Бланксби-Монтгомери для $\ln \mathrm{M}(\alpha)$.

В работах по линейньм формам от логарифмов давно было замечено, что в случае малости $|\ln \alpha|$ по сравнению с $A$ можно получать значительно более сильные результаты. Такая ситуация описывается параметром $E \geqslant A /|\ln \alpha|$. Это равносильно тому, что можно вставить этот параметр в определение числа $A$ и считать $A \geqslant \max \{\ln \mathrm{M}(\alpha), E|\ln \alpha|\}$.

В 1999 г. автор [6] сделал важное наблюдение, а именно - в алгебраическом поле не может быть нескольких мультипликативно независимых малых чисел. Для количественного описания этой ситуации следует ввести более детальные обозначения.

Во-первых, до конца работы у нас будет зафиксировано алгебраическое поле $\mathbb{K} \subseteq \mathbb{C}$ степени $D=[\mathbb{K}: \mathbb{Q}]$, при этом положим $\kappa=1$, если $\mathbb{K} \subseteq \mathbb{R}$, и $\kappa=2$ в противном случае. Имеется $D$ гомоморфизмов $\mathbb{K}$ в $\mathbb{C}: \mathbb{K} \rightarrow \mathbb{K}^{(\bar{\sigma})} \subseteq \mathbb{C}, \sigma=$ $1, \ldots, D$. Мы будем отождествлять $\mathbb{K}=\mathbb{K}^{(1)}$, кроме того, при $\kappa=2$ считаем $\mathbb{K}^{(2)}$ комплексно сопряженным с $\mathbb{K}$.

Во-вторых, для $\alpha \in \mathbb{K}^{*}=\mathbb{K} \backslash\{0\}$ положим

$$
\mathrm{h}(\alpha):=\left.\frac{1}{2 D} \sum_{\sigma=1}^{\infty}|\ln | \alpha\right|_{\sigma}\left|, \quad \mathrm{H}(\alpha):=\frac{D}{\kappa} \mathrm{h}(\alpha), \quad \mathrm{H}^{*}(\alpha):=\frac{1}{2 \kappa} \sum_{\sigma>\kappa}\right| \ln |\alpha|_{\sigma} \mid,
$$

где $|\alpha|_{\sigma}$ означает нормализованное нормирование и будет уточнено в следующем параграфе. Величина $\mathrm{h}(\alpha)$ называется абсолютной логарифмической высотой числа, она равна $\ln \mathrm{M}(\alpha) / d$ и не зависит от поля, в которое помещено число. Выражения $\mathrm{H}(\alpha), \mathrm{H}^{*}(\alpha)$ назовем соответственно логарифмической высотой в поле 
и неполной логарифмической высотой (они зависят также от вложения $\mathbb{K}$ в $\mathbb{C}$ ). Если дополнительно выбрано значение $\ln \alpha \in \mathbb{C}$ и зафиксирован параметр $E>0$, то расширенной логарифмической высотой числа $\alpha$ назовем выражение

$$
\mathrm{H}_{E}^{*}(\alpha):=\max \left\{\mathrm{H}^{*}(\alpha), E|\ln \alpha|\right\} .
$$

Отметим, что в работе [6] вместо параметра $E$ был использован параметр $\theta=$ $1 / E$, стоявший перед компонентой $\mathrm{H}^{*}(\alpha)$. С учетом этого мы и будем приводить результаты работы [6].

В-третьих, нам потребуется еше один параметр. Далее у нас будет зафиксирован набор чисел $\alpha_{1}, \ldots, \alpha_{n} \in \mathbb{K}^{*}$ с выбранньми значениями логарифмов, линейно независимьми над $\mathbb{Z}$. Теперь рассмотрим числа $\beta \in \mathbb{C}$ и их логарифмы, определяемые соотношением

$$
\ln \beta=m_{1} \ln \alpha_{1}+\cdots+m_{n} \ln \alpha_{n} .
$$

Очевидно, при $\mathbf{m}=\left(m_{1}, \ldots, m_{n}\right) \in \mathbb{Z}^{n}$ мы имеем $\beta \in \mathbb{K}^{*}$. Однако могут быть и другие наборы $\mathbf{m}=\left(m_{1}, \ldots, m_{n}\right) \in \mathbb{Q}^{n}$, для которых также $\beta \in \mathbb{K}^{*}$. Обозначим множество таких векторов $\mathbf{m}$ через М. Известно, что при линейной независимости логарифмов множество М будет решеткой, содержашей $\mathbb{Z}^{n}$ как подрешетку конечного индекса. Положим

$$
N=\left[\mathrm{M}: \mathbb{Z}^{n}\right] .
$$

Одним из результатов работы [6] (см. также [7; предложение 5.1]) было

ПРЕДЛОЖЕНИЕ 1.1 (см. [6; теорема 6.2]). Имеет место неравенство

$$
\begin{gathered}
\mathrm{H}_{E}^{*}\left(\alpha_{1}\right) \cdots \mathrm{H}_{E}^{*}\left(\alpha_{n}\right) C_{1.1}^{n} n^{\kappa}\left(\frac{D}{\kappa}\right) \ln \left(\frac{e^{n+4} D}{\kappa}\right)>N \\
C_{1.1}=\frac{e^{\kappa /(1+4 / E)}(1+4 / E)}{2} .
\end{gathered}
$$

Неравенство (1.1) играет двоякую роль. Во-первых, оно дает нетривиальную совместную нижнюю оценку расширенных логарифмических высот мультипликативно независимых чисел, так как всегда $N \geqslant 1$. Во-вторых, оно дает верхнюю оценку индекса указанной выше числовой группы. Оба эти обстоятельства были существенно использованы автором при усилении оценок линейных форм от логарифмов алгебраических чисел [7].

Вскоре в 2000 г. появилась статья Аморозо и Давида [8], в которой было получено важное асимптотическое усиление оценки (1.1).

ПрЕДЛОЖЕНИЕ 1.2 (см. [8; теорема 1.6]). Пусть $k(n):=(n+1)(n+1) !^{n}-n$. Тогда для любых ненулевых мультипликативно независимых алгебраических чисел $\alpha_{1}, \ldots, \alpha_{n} \in \mathbb{K}^{*}$ выполняется неравенство

$$
\mathrm{h}\left(\alpha_{1}\right) \cdots \mathrm{h}\left(\alpha_{n}\right) D \ln ^{n k(n)}(3 D) \geqslant c(n),
$$

где $c(n)>0$ - некоторая константа.

По сравнению с оценкой (1.1) в (1.2) произошли следующие усиления. Во-первых, не требуется выбор $\ln \alpha_{j}$, и, во-вторых, в абсолютной логарифмической высоте происходит деление на $D$, что усиливает оценку в $D^{n}$ раз. Так что при фиксированном $n$ и растущем $D$ совместная оценка высот в неравенстве $(1.2)$ получается лучше, чем в (1.1). 
К достоинствам оценки (1.1) относятся: явный вид со сравнительно небольшими коэффициентами (так, преимущество оценки (1.2) начинает проявляться только при $\left.\ln D>n(n+1) !^{n}\right)$ и наличие верхней оценки индекса, что после статьи Аморозо и Давида выдвигается на первьй план.

В настояшей работе мы снова возврашаемся к оценке типа (1.1). Дело в том, что ее зависимость от параметра $E$ получилась несильной. Хотя константа $C_{1.1}$ в оценке не велика, но ее улучшение с ростом $E$ ограничено, поскольку всегда остается $1+4 / E>1$. Оказывается, при больших значениях $E$ неравенство (1.1) можно заметно усилить.

ТЕОРема 1.3. Пусть дан набор чисел $\alpha_{1}, \ldots, \alpha_{n} \in \mathbb{K}^{*}$ с выбранными значениями логарифмов, линейно независимыми над $\mathbb{Z}$. В действительном случае $(\kappa=1)$ считаем числа $\alpha_{j}$ положстельными, а значения их логарифмов главными. Параметры $A_{1}, \ldots, A_{n}, E, E_{1}, C, \eta>0, J_{1} \in \mathbb{N}$ удовлетворяют условиям

$$
\begin{gathered}
\frac{\mathrm{H}^{*}\left(\alpha_{1}\right)}{A_{1}}+\cdots+\frac{\mathrm{H}^{*}\left(\alpha_{n}\right)}{A_{n}} \leqslant n \\
\max \left| \pm \frac{\ln \alpha_{1}}{A_{1}} \pm \cdots \pm \frac{\ln \alpha_{n}}{A_{n}}\right| \leqslant \frac{n}{E} \\
J_{1} \geqslant \kappa+D \ln \left(J_{1}+1\right), \\
E_{1} e^{E_{1}+1+1 / \kappa} \geqslant 2 E\left(1+\frac{1}{J_{1}-1}\right), \\
C=\frac{n\left(1+1 /\left(J_{1}-1\right)\right)}{2 E_{1}}, \quad \eta=\frac{e^{E_{1}+1+1 / \kappa}}{4 C}, \\
C_{1.3}=C^{n}\left(\frac{n \eta}{E}\right)^{\kappa} .
\end{gathered}
$$

Тогда выполняется неравенство

$$
N \leqslant A_{1} \cdots A_{n} C_{1.3} J_{1}
$$

СлЕДСТВИЕ 1.4. Если в условиях теоремы 1.3 выполняется $\mathrm{H}_{E}^{*}\left(\alpha_{j}\right) \leqslant A_{j}$, $j=1, \ldots, n$, то имеет место оченка (1.8).

СлЕДСТВИЕ 1.5. Пусть

$$
\alpha_{0}, \ldots, \alpha_{n} \in \mathbb{K}^{*}, \quad \operatorname{rank}_{\mathbb{Z}}\left\{\ln \alpha_{0}, \ldots, \ln \alpha_{n}\right\}=n, \quad \mathrm{H}_{E}^{*}\left(\alpha_{j}\right) \leqslant A_{j}, \quad j=0, \ldots, n .
$$

Тогда существует такой ненулевой набор $\mathbf{m}=\left(m_{0}, \ldots, m_{n}\right) \in \mathbb{Z}^{n+1}$, что

$$
m_{0} \ln \alpha_{0}+\cdots+m_{n} \ln \alpha_{n}=0
$$

и в обозначениях теоремы 1.3 выполняются неравенства

$$
\left|m_{j}\right| A_{j} \leqslant A_{0} \cdots A_{n} C_{1.3} J_{1}, \quad j=0, \ldots, n .
$$

В $\S 4$ приводится обсуждение этих результатов и их упрощение. 


\section{§2. Предварительные сведения}

В настоящем параграфе уточняются основные обозначения и приводятся вспомогательные результаты, которые будут использованы при доказательстве основной теоремы 1.3. В целом доказательство теоремы 1.3 практически воспроизводит аналогичное доказательство из работы [6].

Сначала уточним определение нормирований поля $\mathbb{K}$. Для этого пронумеруем простые идеалы $\mathfrak{p}$ из кольца целых чисел поля индексами $\sigma>D$ в порядке возрастания их норм - Norm $\mathfrak{p}$. Тогда для числа $\alpha \in \mathbb{K}^{*}, m=\operatorname{ord}_{\mathfrak{p}}(\alpha), \mathfrak{p}=\mathfrak{p}_{\sigma}$, положим

$$
|\alpha|_{\sigma}:= \begin{cases}\left|\alpha^{(\sigma)}\right|, & 1 \leqslant \sigma \leqslant D \\ (\text { Norm } \mathfrak{p})^{-m}, & \sigma>D\end{cases}
$$

Нормирования, соответствующие комплексно сопряженным $\sigma$, равны, но мы учитываем их оба. Это позволяет не проводить их специальную нормализацию и работать с ними как с обычным модулем. Кроме того, удобство такого учета, а также вышеуказанной нормализации неархимедовских нормирований состоит в формуле произведения без весовых коэффициентов, принимаюшей после логарифмирования вид

$$
\sum_{\sigma=1}^{\infty} \ln |\alpha|_{\sigma}=0, \quad \alpha \in \mathbb{K}^{*},
$$

при этом $|\alpha|_{\sigma} \neq 1$ может быть только для конечного числа индексов $\sigma$.

Далее, приведем необходимые сведения из геометрии чисел. Соответствующие сведения можно найти в [9]. Решеткой мы будем назьвать дискретную подгрупу конечномерного векторного пространства над $\mathbb{R}$. Каждая решетка М имеет целочисленный базис, т.е. $\mathrm{M}=\left\langle\mathbf{a}_{1}, \ldots, \mathbf{a}_{n}\right\rangle_{\mathbb{Z}}$, причем базисные векторы линейно независимы над $\mathbb{R}$. В настоящей работе нам понадобятся только полные решетки, базис которых является базисом всего пространства. Так что в таком базисе пространства векторы решетки будут иметь координаты из $\mathbb{Z}^{n}$. Объем параллепипеда, построенного на базисных векторах решетки, не зависит от выбора базиса и называется обгемом решетки $\operatorname{Vol}(\mathrm{M})$.

Подгруппы решетки сохраняют дискретность и поэтому тоже являются решетками (подрешетками). Если $\Lambda \subseteq \mathrm{M}$ - две полные решетки, то индекс [M : $\Lambda]$ конечен, а из теории групп известно, что выполняется

$$
[\mathrm{M}: \Lambda] \mathbf{a} \in \Lambda, \quad \mathbf{a} \in \mathrm{M}
$$

Имеет место формула

$$
\operatorname{Vol}(\Lambda)=[\mathrm{M}: \Lambda] \operatorname{Vol}(\mathrm{M})
$$

Кроме того, нам потребуется следуюшее утверждение.

ЛЕмма 2.1 (теорема Блихфельдта). Пусть в пространстве задань полная решетка М и компактное тело $\mathscr{W}$. Тогда найдутся различные точки $\mathbf{v}_{0}, \ldots, \mathbf{v}_{J} \in \mathscr{W}$, для которых $\mathbf{v}_{j}-\mathbf{v}_{0} \in \mathrm{M}, j=1, \ldots, J$ (т.е. сравнимые по модулю решетки), и при этом $J \geqslant \operatorname{Vol}(\mathscr{W}) / \operatorname{Vol}(\mathrm{M})$.

Из геометрии выпуклых тел нам потребуется лемма, доказанная в [10] (также приводится в [6] и доказывается с большей степенњю подробности в [7]): 
ЛЕмма 2.2 (см. [10; лемма 5.1]). Пусть в пространстве $\mathbb{R}^{n}$ дано выпуклое 0-симметричное тело $\mathscr{K}$ и линейная форма $\mathbf{f}$ с действительными $(\kappa=1)$ или комплексными $(\kappa=2)$ коэффициентами. Положим

$$
\eta_{0}=\frac{1}{\sup \{\mathbf{f} \cdot \mathbf{w}: \mathbf{w} \in \mathscr{K}\}}, \quad \mathscr{E}(\eta)=\left\{\mathbf{v} \in \mathbb{R}^{n}:|\mathbf{f} \cdot \mathbf{v}| \leqslant \frac{1}{\eta}\right\}, \quad \eta>0
$$

$(\mathbf{f} \cdot \mathbf{v}$ обозначает значение линейной формы $\mathbf{f} н а$ векторе $\mathbf{v})$. Тогда

$$
\operatorname{Vol}(\mathscr{K} \cap \mathscr{E}(\eta)) \geqslant\left(\min \left\{1, \frac{\eta_{0}}{\eta}\right\}\right)^{\kappa} \operatorname{Vol}(\mathscr{K}) .
$$

ЛЕмма 2.3. Пусть $\beta_{1}, \ldots, \beta_{J} \in \mathbb{K}^{*}-$ различные числа с фиксированными значениями логарифмов, $J \geqslant 2 ; z_{1} \in \mathbb{C} ; x_{\sigma} \in \mathbb{R}(\sigma>\kappa)$ - некоторые числа. Пусть с некоторыми $X, Y>0$ выполняются неравенства

$$
\left.\frac{1}{2 \kappa} \sum_{\sigma>\kappa}^{\infty} \max _{1 \leqslant j \leqslant J}\left|x_{\sigma}+\ln \right| \beta_{j}\right|_{\sigma}\left|\leqslant X, \quad \max _{1 \leqslant j \leqslant J}\right| z_{1}+\ln \beta_{j} \mid \leqslant Y .
$$

Тогда имеет место неравенство

$$
\frac{D}{\kappa} \cdot \frac{\ln J}{J-1}+1+\frac{X J}{J-1}-\ln \left(\frac{2(J-1)}{J Y}\right) \geqslant 0
$$

ПРИмЕчАНИЕ. Неравенства (2.4) означают, что числа $\beta_{j}$ близки друг к другу, а при достаточно малых значениях $X, Y$ неравенство (2.5) приближенно имеет вид $(D / \kappa)(\ln J) /(J-1) \geqslant \mathrm{const}>0$ и фактически ограничивает сверху $J-$ количество таких чисел $\beta_{j}$.

ДокАЗАТЕЛЬСТво. Хотя лемма доказана в [6; лемма 7.2], но ввиду краткости доказательства и сильно изменившихся обозначений мы воспроизведем его.

Положим $T=(J-1) / 2$ и рассмотрим функцию

$$
\begin{aligned}
F(z) & =z^{-2 J T}\left(\beta_{1} \cdots \beta_{J}\right)^{-2 T z} \operatorname{det}^{2}\left(\beta_{j}^{z(t-1)}\right)_{j, t=1, \ldots, J} \\
& =z^{-2 J T} \operatorname{det}^{2}\left(\beta_{j}^{z(t-1-T)}\right)_{j, t=1, \ldots, J} \\
& =z^{-2 J T} \operatorname{det}^{2}\left(e^{z(t-1-T)\left(z_{1}+\ln \beta_{j}\right)}\right)_{j, t=1, \ldots, J}
\end{aligned}
$$

(добавить $z_{1}$ можно, так как сумма по $t$ чисел $(t-1-T)$ равна нулю).

Поскольку здесь стоит определитель Вандермонда, по условию мы имеем $\Delta=$ $F(1) \neq 0$. Кроме того, определитель Вандермонда дает множители вида $\left(\beta_{j}^{z}-\beta_{k}^{z}\right)$, $j \neq k$, имеющие нуль при $z=0$. Всего таких множителей $J(J-1)=2 J T$ штук, поэтому $F(z)$ имеет при $z=0$ устранимую особенность.

Для оценки $|F(z)|$ применим неравенство Адамара и условие (2.4):

$$
|F(z)| \leqslant|z|^{-2 T J} J^{J} \exp \left(2 Y|z| \sum_{t=1}^{J}|t-1-T|\right) \leqslant|z|^{-2 T J} J^{J} e^{|z| Y J^{2} / 2}
$$


Теперь рассмотрим окружность $|z|=R=4 T /(J Y)$. Если $R \leqslant 1$, то неравенство (2.5) очевидно выполнено. Если $R>1$, то применение принципа максимума дает

$$
\begin{aligned}
\ln |\Delta| & =\ln |F(1)| \leqslant \max _{|z|=R}|F(z)| \leqslant J \ln J-2 T J \ln |z|+\frac{|z| Y J^{2}}{2} \\
& =J \ln J+J(J-1)-J(J-1) \ln \left(\frac{2(J-1)}{J Y}\right) .
\end{aligned}
$$

Далее, мы имеем $\Delta=F(1) \in \mathbb{K}^{*}$. Неравенство (2.7), а также (2.6) при $z=1$ дают оценку $|\Delta|_{\sigma}, \sigma \leqslant \kappa$. Неравенства, аналогичные (2.6), получаются и для остальных $\sigma$, только для неархимедовских нормирований $(\sigma>D)$ множителя $J^{J}$ не будет:

$$
\begin{array}{ll}
\ln |\Delta|_{\sigma} \leqslant J \ln J+\left.\left(\frac{J^{2}}{2}\right) \max _{1 \leqslant j \leqslant J}\left|x_{\sigma}+\ln \right| \beta_{j}\right|_{\sigma} \mid, & \kappa<\sigma \leqslant D ; \\
\ln |\Delta|_{\sigma} \leqslant\left.\left(\frac{J^{2}}{2}\right) \max _{1 \leqslant j \leqslant J}\left|x_{\sigma}+\ln \right| \beta_{j}\right|_{\sigma} \mid, & \sigma>D .
\end{array}
$$

Сложение неравенств (2.7)-(2.9) (в зависимости от $\sigma$ ) дает, с учетом обозначения (2.4) для $X$ после деления на $\kappa J(J-1)$ и применения формулы произведения (2.1), неравенство (2.5).

\section{§3. Доказательство основных результатов}

ДОКАЗАТЕЛЬСТВО ТЕОРЕМЫ 1.3. Пусть М - решетка из условий теоремы 1.3. Согласно формуле (2.3) с $\Lambda=\mathbb{Z}^{n} \subseteq \mathrm{M}$ мы имеем

$$
\operatorname{Vol}(\mathrm{M})=\frac{\operatorname{Vol}\left(\mathbb{Z}^{n}\right)}{N}=\frac{1}{N} .
$$

Рассмотрим в пространстве $\mathbb{R}^{n}$ линейную форму $\mathbf{f} \cdot \mathbf{v}=v_{1} \ln \alpha_{1}+\cdots+v_{n} \ln \alpha_{n}$ и определим тело $\mathscr{K}$ условиями

$$
\left|w_{j}\right| \leqslant \frac{1}{A_{j}}, \quad j=1, \ldots, n,
$$

и для параметра $\eta$ из условий (1.7)определим тело $\mathscr{K}_{\eta}$ формулой

$$
\mathscr{K}_{\eta}=\mathscr{K} \cap \mathscr{E}(\eta)=\left\{\mathbf{w} \in \mathscr{K}:|\mathbf{f} \cdot \mathbf{w}| \leqslant \frac{1}{\eta}\right\} .
$$

Отметим, что для векторов $\mathbf{w} \in \mathscr{K}$ мы согласно (1.4) имеем

$$
|\mathbf{f} \cdot \mathbf{w}|=\left|w_{1} \ln \alpha_{1}+\cdots+w_{n} \ln \alpha_{n}\right| \leqslant \frac{n}{E},
$$

т.е. для $\eta_{0}$ из леммы 2.2 выполняется $\eta_{0} \leqslant E / n$. Из условий $(1.6),(1.7)$ мы имеем

$$
\frac{\eta}{\eta_{0}} \geqslant \frac{n \eta}{E} \geqslant \frac{E_{1} e^{E_{1}+1+1 / \kappa}}{2 E\left(1+1\left(J_{1}-1\right)\right)} \geqslant 1 .
$$


Лемма 2.2 тогда дает, что

$$
\operatorname{Vol}\left(\mathscr{K}_{\eta}\right) \geqslant\left(\min \left\{1, \frac{\eta_{0}}{\eta}\right\}\right)^{\kappa} \operatorname{Vol}(\mathscr{K}) \geqslant\left(\frac{n \eta}{E}\right)^{-\kappa} \frac{2^{n}}{A_{1} \cdots A_{n}} .
$$

Из формулы (3.1) и теоремы Блихфельта следует существование таких различных векторов $\mathbf{v}_{0}, \ldots, \mathbf{v}_{J} \in \mathscr{W}=\mathscr{K}_{\eta} /(2 C)\left(C\right.$ из (1.7)), что $\mathbf{m}_{j}=\mathbf{v}_{j}-\mathbf{v}_{0} \in \mathrm{M}$, $j=1, \ldots, J$, и

$$
J \geqslant \frac{\operatorname{Vol}(\mathscr{W})}{\operatorname{Vol}(\mathrm{M})} \geqslant\left(\frac{n \eta}{E}\right)^{-\kappa} \frac{N C^{-n}}{A_{1} \cdots A_{n}} .
$$

Теперь воспользуемся леммой 2.3, взяв

$$
\begin{aligned}
z_{\sigma} & =v_{1,0} \ln \alpha_{1}^{(\sigma)}+\cdots+v_{n, 0} \ln \alpha_{n}^{(\sigma)}, & & \sigma \leqslant \kappa, \\
x_{\sigma} & =v_{1,0} \ln \left|\alpha_{1}\right|_{\sigma}+\cdots+v_{n, 0} \ln \left|\alpha_{n}\right|_{\sigma}, & & \sigma=1,2, \ldots, \\
\ln \beta_{j} & =m_{1, j} \ln \alpha_{1}+\cdots+m_{n, j} \ln \alpha_{n}, & & j=1, \ldots, J .
\end{aligned}
$$

Покажем, что условия (2.4) выполнены с

$$
X=\frac{n}{2 C}, \quad Y=\frac{1}{2 C \eta}
$$

Из определения тела $\mathscr{K}_{\eta}$ и векторов $\mathbf{v}_{j}, \mathbf{m}_{j}$ мы имеем

$$
\begin{aligned}
\left|z_{1}+\ln \beta_{j}\right| & =\left|\mathbf{f} \cdot\left(\mathbf{v}_{0}+\mathbf{m}_{j}\right)\right|=\left|\mathbf{f} \cdot \mathbf{v}_{j}\right|=\left|v_{1, j} \ln \alpha_{1}+\cdots+v_{1, n} \ln \alpha_{n}\right| \\
& \leqslant \frac{1}{2 C \eta}=Y, \\
\left.\left|x_{\sigma}+\ln \right| \beta_{j}\right|_{\sigma} \mid & =\left.\left|v_{1, j} \ln \right| \alpha_{1}\right|_{\sigma}+\cdots+v_{n, j} \ln \left|\alpha_{n}\right|_{\sigma} \mid \\
& \leqslant \frac{1}{2 C}\left(\frac{\left.|\ln | \alpha_{j}\right|_{\sigma} \mid}{A_{1}}+\cdots+\frac{\left.|\ln | \alpha_{n}\right|_{\sigma} \mid}{A_{n}}\right)
\end{aligned}
$$

а за счет условия (1.3) на параметры $A_{j}$ выполняется

$$
\begin{aligned}
\left.\frac{1}{2 \kappa} \sum_{\sigma>\kappa}^{\infty} \max _{1 \leqslant j \leqslant J}\left|x_{\sigma}+\ln \right| \beta_{j}\right|_{\sigma} \mid & \leqslant \frac{1}{2 \kappa} \sum_{\sigma>\kappa}^{\infty} \frac{1}{2 C}\left(\frac{\left.|\ln | \alpha_{1}\right|_{\sigma} \mid}{A_{1}}+\cdots+\frac{\left.|\ln | \alpha_{n}\right|_{\sigma} \mid}{A_{n}}\right) \\
& \leqslant \frac{1}{2 C}\left(\frac{\mathrm{H}_{E}^{*}\left(\alpha_{1}\right)}{A_{1}}+\cdots+\frac{\mathrm{H}_{E}^{*}\left(\alpha_{n}\right)}{A_{n}}\right) \leqslant \frac{n}{2 C}=X .
\end{aligned}
$$

Проверим, что числа $\beta_{j}$ различны. Отметим, что вследствие линейной независимости логарифмов $\ln \alpha_{1}, \ldots, \ln \alpha_{n}$ равенство чисел $\beta_{j}$ возможно только в случае $\ln \beta_{j}-\ln \beta_{\kappa}=2 \pi i \mu, \mu \in \mathbb{Z}, \mu \neq 0$. Но у нас

$$
0 \neq\left|\ln \beta_{j}-\ln \beta_{k}\right| \leqslant 2 Y=\frac{1}{C \eta} \leqslant \frac{4}{e^{E_{1}+1+1 / \kappa}}<2 \pi
$$

следовательно, числа $\beta_{j}$ различны и применение леммы 2.3 дает выполнение неравенства (2.5).

Теперь с учетом неравенства (3.2) для доказательства теоремы 1.3 достаточно получить оценку $J \leqslant J_{1}$. 
Если $J \leqslant J_{1}$, то требуемая оценка уже есть. Так что можно считать $J \geqslant$ $J_{1}+1 \geqslant 2$ и применять лемму 2.3. Неравенство $(2.5)$ после умножения на $(J-1)$, подстановки выражений для $X, Y, \eta$ и приведения подобных членов примет вид

$$
\frac{D}{\kappa} \ln J-(J-1) E_{1}+\frac{n J}{2 C}+(J-1) \ln \left(1+\frac{1}{J-1}\right) \geqslant 0 .
$$

После подстановки $C$ из формулы $(1.7)$ и обозначения $\varepsilon_{1}=1 /\left(J_{1}-1\right)$ получаем следующее неравенство

$$
\frac{D}{\kappa} \ln J-\frac{1+\left(E_{1} \kappa+1\right) \varepsilon_{1}}{\left(1+\varepsilon_{1}\right) \kappa} J+E_{1}+\frac{1}{\kappa}+(J-1) \ln \left(1+\frac{1}{J-1}\right) \geqslant 0 .
$$

Теперь можно заменить $(J-1) \ln (1+1 /(J-1))$ на 1 с усилением неравенства, а $\varepsilon_{1}$ - на меньшее число $\varepsilon=1 /(J-1)$ также с усилением неравенства. После умножения на $\kappa$ и приведения подобных членов это дает неравенство

$$
J-1-\kappa-D \ln J<0 .
$$

Покажем, что из неравенства (3.3) также следует $J<J_{1}+1$.

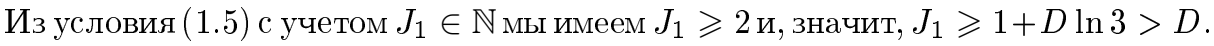
При $J=J_{1}+1>D$ левая часть неравенства (3.3) в силу условия (1.5) неотрицательна, а с ростом $J$ она возрастает, поэтому при $J \geqslant J_{1}+1$ неравенство (3.3) неверно.

Следствие 1.4 сразу получается из теоремы 1.3 , поскольку условия (1.3), (1.4) вьполняются в силу определения расширенной логарифмической высоты.

ДОКАЗАТЕЛЬСТВо СЛЕДСТВИЯ 1.5. Рассмотрим какой-нибудь один индекс, скажем $j=0$. Если $\operatorname{rank}_{\mathbb{Z}}\left\{\ln \alpha_{1}, \ldots, \ln \alpha_{n}\right\}<n$, то $\ln \alpha_{0}$ в линейном соотношении (1.9) не участвует, т.е. $m_{0}=0$, и для него неравенство тривиально. Если $\operatorname{rank}_{\mathbb{Z}}\left\{\ln \alpha_{1}, \ldots, \ln \alpha_{n}\right\}=n$, то согласно формуле (2.2) имеется целочисленное соотношение между логарифмами с $m_{0}^{\prime}=N$, при этом применимо следствие 1.4 , в котором оценка (1.8) индекса $N$ дает оценку (1.10) для $j=0$, так как множитель $A_{0}$ сокращается. Аналогичные рассуждения для остальных $j$ также дают оценку $m_{j}^{\prime}$ вида (1.10), где пока что для каждого $j$ может быть свой вектор $\mathbf{m}$.

Поскольку по условию $\operatorname{rank}_{\mathbb{Z}}\left\{\ln \alpha_{0}, \ldots, \ln \alpha_{n}\right\}=n$, соотношение между векторами единственно с точностью до множителя из $\mathbb{Z}$, сокрашение которого сохраняет оценки коэффициентов. Так что в неравенстве (1.10) оценка при различных $j$ относится к одному и тому же вектору $\mathbf{m}$, если считать его примитивным.

\section{§4. Обсуждение результатов}

В отличие от вполне явной оценки (1.1) основная теорема 1.3 содержит некоторую неопределенность в приложениях, поскольку в ее условиях присутствуют параметры, значения которых требуют подгонки, причем их выбор неочевиден и может сильно повлиять на качество оценки (1.8). Это оставлено по той причине, что точные формулы для оптимальных значений параметров были бы слишком 
громоздки. В важных частных случаях подгонку можно сделать численно. Ниже мы приведем огрубленный выбор параметров.

Смысл параметров $A_{1}, \ldots, A_{n}, E$, определяющих вьполнение условий (1.3), (1.4), был объявлен ранее и виден в следствии 1.4. Отметим только, что поскольку $\mathrm{H}^{*}(\alpha) \leqslant \mathrm{H}(\alpha)$, в формулировках результатов можно заменить $\mathrm{H}^{*}(\alpha)$ на $\mathrm{H}(\alpha)$ с некоторым ослаблением утверждений. Так, в случае, когда $\mathbb{K}=\mathbb{Q}$ и $\alpha=p-$ простое число, мы имеем $\mathrm{H}(\alpha)=\ln p, \mathrm{a}^{*}(\alpha)=(\ln p) / 2$, т.е. получаем выигрыш в два раза.

Ниже мы приведем упрошенные рабочие формулы для параметров $E_{1}, J_{1}$, считая значения $A_{1}, \ldots, A_{n}, E$ фиксированными. При этом отметим, что наилучшая ситуация в условиях (1.5), (1.6) не обязательно будет при выполнении в них равенств. Рассмотрим сначала главное усиление настоящей статьи - случай большого значения $E$.

Лемма 4.1. Пусть $\varepsilon_{1}, E_{1}^{*}, E_{0}, E_{0}^{\prime}$ определяются равенствами

$$
\begin{gathered}
\varepsilon_{1}=\frac{1}{J_{1}-1}, \quad E_{1}^{*} e^{E_{1}^{*}+1+1 / \kappa}=2 E\left(1+\varepsilon_{1}\right), \\
E_{0}=2 E e^{-1-1 / \kappa}\left(1+\varepsilon_{1}\right), \quad E_{0}^{\prime}=E_{0}\left(1-\frac{1}{e}\right)^{-1} .
\end{gathered}
$$

Тогда при $E_{0}>1$ выполняется двусторонняя оценка

$$
\ln \left(\frac{E_{0}}{\ln E_{0}}\right)<E_{1}^{*} \leqslant \ln \left(\frac{E_{0}^{\prime}}{\ln E_{0}^{\prime}}\right) .
$$

ДоКАЗАТЕЛЬСТво. Прологарифмируем неравенство (1.6) и подставим в него $E_{1}=\ln \left(E_{0}^{\prime} / \ln E_{0}^{\prime}\right)$ :

$$
\begin{aligned}
E_{1}+ & \ln E_{1}+1+\frac{1}{\kappa}=\ln \left(\frac{E_{0}^{\prime}}{\ln E_{0}^{\prime}}\right)+\ln \left(\ln \left(\frac{E_{0}^{\prime}}{\ln E_{0}^{\prime}}\right)\right)+1+\frac{1}{\kappa} \\
& =\ln \left(2 E e^{-1-1 / \kappa}\left(1-\frac{1}{e}\right)^{-1}\left(1+\varepsilon_{1}\right)\right)+\ln \left(1-\frac{\ln \ln E_{0}^{\prime}}{\ln E_{0}^{\prime}}\right)+1+\frac{1}{\kappa} \\
& \geqslant \ln \left(2 E\left(1+\varepsilon_{1}\right)\right) .
\end{aligned}
$$

Значит, условие (1.6) выполнено, следовательно, $E_{1}^{*} \leqslant \ln \left(E_{0}^{\prime} / \ln E_{0}^{\prime}\right)$. Аналогично доказывается другое неравенство.

Таким образом, $E_{1}^{*} \sim \ln \left(E_{0} / \ln E_{0}\right) \sim \ln E, E \rightarrow \infty$. При выборе $E_{1}=E_{1}^{*}$ мы имеем $n \eta / E=1$, так что зависимость оценки (1.8) от $E$ проявляется в параметре $C$ и имеет вид $E_{1}^{-n} \sim(\ln E)^{-n}$, т.е. неограниченно улучшается с ростом $E$. Это основное отличие теоремы 1.3 от предложения 1.1 .

Случай небольших значений $E$ фактически был рассмотрен в работе [6]. Здесь ситуация оказывается совершенно иной, чем для больших $E$. Дело в том, что общая зависимость оценки (1.8) от параметра $E_{1}$ с учетом его вхождения в $\eta$ имеет вид $e^{\kappa E_{1}} / E_{1}^{n-\kappa}$, поэтому решение небольшой задачи на экстремум показьвает, что выгоднее брать $E_{1} \geqslant(n-\kappa) / \kappa\left(\right.$ для простоты $\left.E_{1} \geqslant n / \kappa\right)$, а не $E_{1}=E_{1}^{*}$. При доказательстве теоремы 1.3 этому соответствует случай, когда для получения тела $\mathscr{K}_{\eta}$ от параллелограмма $\mathscr{K}$ приходится отсекать углы. 
При $E_{1}^{*} \leqslant n / \kappa$ выбор $E_{1}=n / \kappa$ допустим, так как дает выполнение условия (1.6), и мы получаем $e^{\kappa E_{1}} / E_{1}^{n-\kappa}=(n / \kappa)^{\kappa} e^{n} \kappa^{n} / n^{n}$, где главное - это появление в знаменателе факториального множителя $n^{n}$ по сравнению с выбором $E_{1}=E_{1}^{*}$. Это усиление было впервые получено автором в работе [10] при исследовании линейных форм от логарифмов алгебраических чисел. В предложении 1.1 этот фактор тоже был учтен. Так что для простоты можно считать

$$
E_{1} \geqslant \max \left\{\frac{n}{\kappa}, E_{1}^{*}\right\} .
$$

Некоторое усиление оценки (1.8) можно получить и за счет правильного выбора параметра $J_{1}$, условие $(1.5)$ для которого также требует расшиирровки.

ЛЕМма 4.2. Условие (1.5) выполняется при

$$
J_{1} \geqslant\left[J_{1}^{*}+1\right], \quad J_{1}^{*}:=\kappa+D \ln (1+\kappa+1.2 D \ln (e D)) .
$$

ДоКАЗАТЕЛЬСТвО. Пусть $J_{1}$ удовлетворяет условию (4.3). Тогда для каждого целого $D \leqslant 100$ и $1 \leqslant \kappa \leqslant \min \{2, D\}$ неравенство (1.5) проверяется численно. При $D \geqslant 100$ ввиду монотонности достаточно проверить условие (1.5) при $J_{1}=J_{1}^{*}$. С учетом этого мы имеем

$$
\begin{gathered}
D \ln (1+\kappa+1.2 D \ln (e D))<D \ln \left(e^{0.2} D \ln (e D)\right)<D \ln \left(e^{1.2} D^{1.2}\right)=1.2 D \ln (e D) \\
J_{1}^{*}-\kappa-D \ln \left(J_{1}^{*}+1\right)=D \ln \left(\frac{1+\kappa+1.2 D \ln (e D)}{1+\kappa+D \ln (1+\kappa+1.2 D \ln (e D))}\right)>0
\end{gathered}
$$

Отметим также, что параметр $J_{1}$ присутствует в оценке $(1.7)$ как непосредственно, так и косвенно через константу $C$, входящую также в $\eta$, окончательно в виде $\left(1+1 /\left(J_{1}-1\right)\right)^{n-\kappa} J_{1}$. Решение простой оптимизационной задачи показывает, что выгоднее брать $J_{1} \geqslant n-\kappa$. Соединение с неравенством (4.3) приводит к упрощенному выбору

$$
J_{1} \geqslant D \ln \left(e^{n+2} D \ln (e D)\right) .
$$

Отметим, что сначала следует выбирать значение $J_{1}$ согласно (4.4), а затем $E_{1}$ согласно оценке (4.2), причем увеличение значения $J_{1}$ улучшает значение $E_{1}^{*}$, поскольку уменьшается $\varepsilon_{1}$ из формул (4.1).

Перейдем к рассмотрению следствия 1.5. Впервые явную оценку коэффициентов в линейном соотношении между логарифмами алгебраических чисел вида (1.10), правда со значительно худшей константой $C_{1.3}$, получил Вальдшмидт [11]. Это было сделано с использованием только методов геометрии чисел. Позднее автор показал [12], что более широкая задача получения совместной оценки для нескольких независимых соотношений вида (1.9) может быть сведена к теореме Минковского о последовательных минимумах. К сожалению, в формулировке соответствующей теоремы был пропущен множитель, имеющийся в теореме Минковского и равньй объему некоторого тела. Эта ошибка была устранена в работе Бертрана [13]. Явный пригодный для применения вид этой оценки был получен автором в работе [7]: если $\operatorname{rank}_{\mathbb{Z}}\left\{\ln \alpha_{1}, \ldots, \ln \alpha_{n}\right\}=r<n$ (нумерация индексов в работе [7] была от 1 до $n$ ), то найдутся $n-r$ линейно независимых векторов 
$\mathbf{m}_{i}=\left(m_{i, 1}, \ldots, m_{i, n}\right) \in \mathbb{Z}^{n}, i=1, \ldots, n-r$, удовлетворяющих условию $(1.9)$ и неравенству (неравенство (21.5) из [7])

$$
\prod_{i=1}^{n-r} \max _{1 \leqslant j \leqslant n}\left\{\left|m_{i, j}\right| A_{j}\right\} \leqslant 4 \sqrt{n}\left(n D^{3} \ln ^{2}(e D)\right)^{r / 2} A_{1} \cdots A_{n}
$$

где подразумевалось, что $A_{j} \geqslant \max \left\{D \mathrm{~h}\left(\alpha_{j}\right),\left|\ln \alpha_{j}\right|, 0.16\right\}$.

Оценка применима и к случаю единственного с точностью до множителя соотношения между логарифмами $(r=n-1)$. Сравнение показывает, что здесь преимушество оценки (1.10) проявляется в следуюшем: константа $C_{1.3}$ практически не зависит от степени поля $D$, имеет показательный (а не факториальный) рост в зависимости от количества логарифмов $n$, а также улучшается с ростом параметра $E$.

Отметим, что оценка (1.10) была получена совместньми методами теории трансцендентных чисел и геометрии чисел, а собственно оценка коэффициентов опирается на простой факт (2.2) из теории групп.

Правда, случай наличия нескольких зависимостей между логарифмами, учитываемый в оценке (4.5), в настоящей статье не рассматривается.

\section{Список литературы}

1. Lehmer D. H. Factorization of certain cyclotomic functions // Ann. of Math. (2). 1933. V. 34. № 2. P. 461-479.

2. Smyth C. J. On the product of the conjugates outside the unit circle of an algebraic integer // Bull. London Math. Soc. 1971. V. 3. P. 169-175.

3. Blanksby P. E., Montgomery H. L. Algebraic integers near the unit circle // Acta Arith. 1971. V. 18. P. $355-369$.

4. Dobrowolski E. On a question of Lehmer and the number of irreducible factors of a polynomial // Acta Arith. 1979. V. 34. P. 391-401.

5. Voutier P. M. An effective lower bound for the height of algebraic numbers // Acta Arith. 1996. V. 74. P. 81-95.

6. Матвеев E. M. О последовательных минимумах расширенной логариффической высоты алгебраических чисел // Матем. сб. 1999. Т. 190. № 3. С. 89-108.

7. Матвеев E. M. Явная нижняя оценка однородной рационалной линейной формы от логарифмов алгебраических чисел. II // Изв. РАН. Сер. матем. 2000. Т. 64. №6 C. $125-180$

8. Amoroso F., David S. Le problème de Lehmer en dimension supériore // J. Reine Angew. Math. 1999. V. 513. P. 145-179.

9. Касселс Дж. В. С. Введение в геометрию чисел. М.: Мир, 1965.

10. Матвеев E. M. Явная нижняя оценка однородной рациональной линейной формы от логарифмов алгебраических чисел // Изв. РАН. Сер. матем. 1998. Т. 62. № 4. С. 81-136.

11. Waldschmidt M. A lower bound for linear forms in logarithms // Acta Arith. 1980. V. 37. P. 257-283.

12. Матвеев E. M. О линейных и мультипликативных соотношениях // Матем. сб. 1993. T. 184. № 4. C. 23-40.

13. Bertrand D. Duality on tori and multiplicative dependence relations // J. Austral. Math. Soc. Ser. A. 1997. V. 62. P. 198-216. 